\title{
Association of elevated fasting glucose with lower patency and increased major adverse limb events among patients with diabetes undergoing infrapopliteal balloon angioplasty
}

\author{
Satinder Singh ${ }^{1}$, Ehrin J Armstrong ${ }^{2}$, Walid Sherif ${ }^{1}$, Bejan Alvandi ${ }^{1}$, Gregory G Westin ${ }^{1}$, \\ Gagan D Singh ${ }^{1}$, Ezra A Amsterdam ${ }^{1}$, and John R Laird ${ }^{1}$ \\ ${ }^{1}$ Division of Cardiovascular Medicine and the Vascular Center, UC Davis Medical Center, \\ Sacramento, CA, USA \\ ${ }^{2}$ Division of Cardiology, University of Colorado and VA Eastern Colorado Healthcare System, \\ Denver, CO, USA
}

\begin{abstract}
Diabetes mellitus (DM) is a significant risk factor for loss of patency after endovascular intervention, but the contribution of glycemic control to infrapopliteal artery patency among patients with DM is unknown. All percutaneous infrapopliteal interventions among patients with DM from 2006 to 2013 were reviewed and pre-procedure fasting blood glucose (FBG) was recorded. The primary endpoint was primary patency at 1 year as determined by duplex ultrasound. A total of 309 infrapopliteal lesions in 149 patients with DM were treated with balloon angioplasty during the study period. The median FBG was $144 \mathrm{mg} / \mathrm{dL}$. At 1 year, the rate of primary patency was $16 \%$ for patients with FBG above the median, compared to $46 \%$ for patients with FBG below the median (hazard ratio (HR) 1.82 for FBG $\geq 144, p=0.005$ ). Amputation rates at 1 year trended higher among patients with high versus low FBG $(24 \%$ vs $15 \%, p=0.1)$. One year major adverse limb event rates were also higher for patients with high versus low FBG (35\% vs $23 \%, p=0.05$ ). Although patients with high FBG were more likely to have insulin-requiring DM ( $73 \%$ vs $50 \%, p=0.003$ ) the association of high FBG with loss of primary patency remained significant even after adjusting for insulin use as well as other lesion-specific characteristics (adjusted HR 1.8, 95\% CI 1.2-2.8). In conclusion, high fasting blood glucose at the time of infrapopliteal balloon angioplasty is associated with significantly decreased primary patency and may also be a risk factor for major adverse limb events among patients with a threatened limb.
\end{abstract}

\section{Keywords}

balloon angioplasty; critical limb ischemia; diabetes; fasting blood glucose; percutaneous intervention; peripheral artery disease

\footnotetext{
Corresponding author: John R Laird, Medical Director of the Vascular Center, UC Davis Medical Center, 4860 Y Street, Suite 3400, Sacramento, CA 95817, USA, john.laird@ucdmc.ucdavis.edu.

Declaration of conflicting interest

Dr Laird is a consultant for Boston Scientific, Covidien, Abbott, Bard, and Medtronic. All other authors have reported that they have no relationships relevant to the contents of this paper to disclose.

Reprints and permissions: sagepub.co.uk/journalsPermissions.nav
} 


\section{Introduction}

Peripheral artery disease (PAD) affects over 12 million people in the United States and approximately $20-30 \%$ of these patients have diabetes. ${ }^{1}$ Diabetes leads to endothelial dysfunction, vascular smooth muscle cell proliferation, and platelet activation. ${ }^{2}$ All of these factors predispose to increased atherosclerosis and impaired wound healing. Patients with diabetes and concomitant PAD are more likely to have infrapopliteal atherosclerotic disease and are more likely to present with ischemic ulcers or gangrene. ${ }^{3,4}$ Diabetics are up to 15 times more likely than non-diabetics to suffer a major amputation. ${ }^{5}$ Diabetes is also associated with decreased primary patency following endovascular interventions. 6,7

The contribution of glycemic control to cardiovascular outcomes in patients with diabetes remains controversial. While intensive glycemic control has not reduced rates of major cardiovascular events in large trials, ${ }^{8,9}$ glycemic control may be associated with improved limb outcomes and vessel patency in patients undergoing endovascular intervention. ${ }^{10}$ This question has been studied primarily in percutaneous coronary interventions (PCI), where some studies have reported that an elevated hemoglobin A1c (HbA1c) is associated with restenosis or target lesion revascularization. ${ }^{11,12}$ Other studies have also reported that fasting glucose levels are a better predictor of restenosis following PCI than HbA1c. ${ }^{13}$ No study, however, has investigated the association of glucose control with outcomes after infrapopliteal balloon angioplasty among patients with diabetes. Because infrapopliteal balloon angioplasty has overall low rates of primary patency, a better understanding of the relationship between hyperglycemia and patency may help identify interventions to minimize target lesion occlusion among diabetic patients.

The purpose of this study was to analyze the association between pre-procedural fasting blood glucose (FBG) and subsequent patency in a cohort of patients with diabetes undergoing infrapopliteal balloon angioplasty for limb salvage. We hypothesized that lower pre-procedural FBG would be associated with improved primary patency and improved limb outcomes.

\section{Methods}

The PAD-UCD (University of California at Davis) Registry consists of all patients with a clinical diagnosis of PAD who underwent diagnostic peripheral angiography or therapeutic endovascular intervention at the University of California Davis Medical Center between 1 June 2006 and 1 June 2013. During this timeframe, three vascular surgeons and one interventional cardiologist performed the procedures. At the time of data extraction, 1146 patients and 1789 procedures were included in the overall registry.

\section{Data collection and definitions}

After approval by the Institutional Review Board at University of California Davis Medical Center, baseline data were collected from review of electronic medical record documentation and procedure notes. Pre- and post-procedure clinical notes and the admission history and physical documentation were used to identify clinical presentation as well as post-procedure 
outcomes and medical management. This information was entered into a pre- specified case report form with standardized data entry, as previously described. ${ }^{14}$

During the study period, 149 patients with diabetes presented with critical limb ischemia (CLI) or acute limb ischemia (ALI) and underwent infrapopliteal interventions. None of these patients had evidence of active systemic infection at the time of endovascular intervention. Because the majority of these procedures were performed for limb salvage, inflow procedures i.e. iliac or superficial femoral/popliteal artery interventions were performed when necessary to establish straight-line flow to the threatened limb. CLI was defined as Rutherford category 4-6 disease (defined as ischemic rest pain, minor tissue loss, or major tissue loss, respectively) based on review of clinic notes, history and physical examination, and hospital discharge summaries. Rutherford category 5 disease was classified as a non-healing lower-extremity ulcer with minor tissue loss, while Rutherford category 6 disease was defined as major tissue loss extending beyond the transmetatarsal level. ${ }^{15}$ Acute limb ischemia was defined as ischemic rest pain with a duration of less than 2 weeks. ${ }^{16}$

FBG was recorded from the basic metabolic panel (BMP) on the day of the procedure. If there was no fasting BMP available on the day of the procedure $(n=48)$, then a FBG was obtained from laboratory values up to 2 days prior or 2 days post procedure. If no FBG was available then the procedure was excluded from analysis. There was no significant difference in FBG values for patients with pre-procedural versus post-procedural laboratory data. The most recent $\mathrm{HbA1c}$ value available within 3 months pre-procedure was also recorded. Ankle-brachial pressure index (ABI) and toe-brachial index (TBI) measurements and duplex ultrasonography (DUS) were obtained in patients before endovascular treatment and at follow-up. A target lesion was defined as endovascular intervention to the tibioperonal trunk, and peroneal, anterior tibial, or posterior tibial artery. Each artery was considered a single target lesion. Quantitative angiography was performed on all target lesions to evaluate the pre- and post-intervention percent diameter stenosis, lesion length, and reference vessel diameter. Chronic occlusions were defined as complete arterial occlusion with absence of antegrade flow. Procedural success was defined as $<30 \%$ stenosis at the conclusion of the procedure without major adverse event. Two cardiologists who were unaware of the FBG values reviewed all angiographic images to verify lesion morphology, calcification, and status of the distal run-off vessels.

Patients were routinely seen 30 days after the revascularization procedure. The follow-up visit included an assessment of the patient's clinical improvement as well as interval ABI, TBI, and DUS examination. Further postoperative follow-up was then conducted every 3 months during the first postoperative year and every 6-12 months thereafter. Target lesion patency was evaluated by DUS at 0-30 days, 4-6 months, 9-12 months, and every 6 months thereafter. Loss of primary patency was defined as the presence of $>50 \%$ stenosis at the treatment site by quantitative angiography, DUS peak systolic velocity ratio $>2.0$, or the need for target lesion revascularization (TLR). TLR was defined as any repeat endovascular intervention or surgical bypass to the intervened lesion. Primary assisted patency was defined as patency after treatment for restenosis, and secondary patency was defined as overall patency after restenosis or occlusion. Major amputation was defined as any amputation above the level of the ankle joint. A major adverse limb event (MALE) was 
defined as major amputation, new bypass graft placement, surgical graft revision, thrombectomy, or thrombolysis. ${ }^{17}$

\section{Outcomes}

The primary endpoint was primary patency. Secondary endpoints included primary assisted patency, secondary patency, major amputation, and MALE.

\section{Data analysis}

We performed a post-hoc analysis on 149 diabetic patients in the PAD-UCD Registry who had presented with CLI or ALI and underwent infrapopliteal interventions. Mean values with standard deviation were used to describe continuous variables, and numerical values (percentages) were used for categorical variables. Continuous variables were compared using the Mann-Whitney test. Categorical values were compared by the Fisher's exact test. Freedom from binary restenosis and freedom from occlusion were analyzed with the use of Kaplan-Meier survival estimates and the log-rank test. Univariate analysis was used to identify predictors of patency after infrapopliteal balloon angioplasty. A multivariable cox regression model was developed to assess the relationship between FBG and target lesion patency. Patient variables known to be associated with restenosis, including insulin use, chronic kidney disease, dialysis, and statin use were automatically forced into the model. Lesion-specific characteristics that increase the risk of restenosis, including lesion length and chronic total occlusion, were also automatically included. Generalized estimating equations were used to account for patient clustering in situations of multiple target lesions per procedure. The value for statistical significance was $<0.05$. Analyses were conducted using STATA Version 11.2 (STATA Corporation, College Station, TX, USA). All authors had full access to and take full responsibility for the integrity of the data. All authors have read and agree to the manuscript as written.

\section{Results}

During the study period, 149 patients with diabetes underwent balloon angioplasty to 309 infrapopliteal lesions. FBG values at the time of the procedure ranged from 55 to 459 $\mathrm{mg} / \mathrm{dL}$, with a mean value of $165 \pm 80 \mathrm{mg} / \mathrm{dL}$ and a median value of $144 \mathrm{mg} / \mathrm{dL}$ (interquartile range $109-213 \mathrm{mg} / \mathrm{dL}$ ). Patients were divided into two groups based on pre-procedure FBG below (low FBG group) or above (high FBG group) the median FBG value.

The baseline patient characteristics are presented in Table 1. There was no difference between groups in age, sex, or other risk factors for atherosclerosis. The baseline rate of statin use was also similar between groups (71\% for high FBG group vs $66 \%$ for low FBG group, $p=0.6)$. Patients with a high FBG were more likely to have DM requiring insulin therapy $(73 \%$ vs $50 \%, p=0.003)$, and also had a higher $\mathrm{HbA1c}(8.4 \%$ vs $7.6 \%, p=0.04)$. There was no significant difference in Rutherford classification or pre-procedure ABI values between groups.

The angiographic characteristics of the target lesions are summarized in Table 2. The most frequent target vessel was the anterior tibial artery in both groups, and the mean lesion length was also similar for the high versus low FBG groups ( $94 \pm 68$ vs $92 \pm 74 \mathrm{~mm}, p=0.9$ ). 
There was also no difference between groups in vessel diameter, prevalence of chronic occlusion, or extent of lesion calcification. Six patients (8\%) in each group had concomitant procedures performed to inflow iliac or superficial femoral artery stenosis. The procedural success rate was similar between groups ( $94 \%$ vs $91 \%, p=0.3$ ). Distal embolization occurred during treatment of $13(4 \%)$ target lesions; all of these cases were managed successfully with thrombectomy and restoration of distal flow. Post-procedure ABI values were also similar for patients in the low versus high FBG groups ( 0.79 vs $0.73, p=0.2)$.

During follow-up, a total of 27 patients underwent repeat angiography with intervention to 45 lesions. Forty-four of the target lesion interventions were triggered by a persistent wound, while one case was performed on an asymptomatic patient with DUS evidence of occlusion of the last remaining run-off vessel. One year post-procedure, the Kaplan-Meier estimated primary patency was $16 \%$ for patients with FBG above the median, compared to $46 \%$ for patients in the low FBG group ( $p=0.005$, Figure 1). Patients with high FBG also had significantly lower rates of primary assisted patency ( $p=0.001$, Figure 2 ) and secondary patency during 1 -year follow-up ( $p=0.004$, Figure 3 ). Analysis of FBG by quartiles also showed a significant dose-effect of FBG and the likelihood of target lesion patency, with primary patency of $49 \%$ for patients in the first quartile, and primary patency of only $11 \%$ for patients in the fourth quartile ( $p=0.02$ for trend, Figure 4 ). There was no significant association between $\mathrm{HbA} 1 \mathrm{c}$ and rates of primary patency (hazard ratio(HR) 1.15, 95\% CI $0.72-1.87$ for HbA1c $>7$ and loss of primary patency).

Amputation rates at 1 year trended higher among patients with high versus low FBG (24\% vs $15 \%, p=0.1$ ). One-year MALE rates were significantly higher for patients with high versus low FBG ( $35 \%$ vs $23 \%, p=0.05$; Figure 5), due primarily to increased rates of both surgical bypass and major amputation among patients with high FBG. There was no significant association between FBG values and overall mortality, amputation-free survival, or rates of major adverse cardiovascular events.

On multivariable analysis (Table 3), the association of high FBG with loss of primary patency remained significant even after adjusting for insulin use as well as lesion length, presence of chronic occlusion, statin use, glomerular filtration rate, and dialysis status (adjusted HR for high FBG 1.8, 95\% CI 1.2-2.8). Applying the same multivariable model to analysis of primary assisted and secondary patency also showed that high FBG at the time of infrapopliteal balloon angioplasty was associated with decreased primary assisted patency (adjusted HR for high FBG 2.0, 95\% CI 1.2-3.1) and secondary patency (adjusted HR 1.8, 95\% CI 1.1-3.0). High FBG also remained associated with an increased rate of subsequent MALE (Table 4) in a multivariable model (adjusted HR 2.3, 95\% CI 1.1-5.2).

\section{Discussion}

To our knowledge, this is the first study to evaluate the influence of pre-procedural glycemic control on outcomes of infrapopliteal balloon angioplasty. We note three major findings. First, we found that elevated FBG was associated with significantly lower primary patency rates at 1 year. Second, MALEs were also significantly higher in the high FBG group. Third, 
FBG was independently associated with loss of primary patency and MALEs even after adjusting for insulin use and other lesion-specific characteristics.

Although diabetes is a risk factor for development of PAD, the association of glycemic control with cardiovascular outcomes remains controversial. Poor glycemic control has been associated with higher prevalence of $\mathrm{PAD}^{18}$ and risk of adverse outcomes, including the need for lower extremity bypass surgery, amputation or death. ${ }^{19}$ In addition, poor glycemic control is also associated with worse outcomes following both vascular surgery ${ }^{20}$ and endovascular ${ }^{6,21}$ procedures. Takahara et al. ${ }^{21}$ studied 278 patients who underwent balloon angioplasty for CLI and found that diabetes and poor glycemic control was each associated with higher rates of major amputations. Diabetes was also associated with lower rates of primary patency after percutaneous interventions. DeRubertis et al. ${ }^{6}$ retrospectively studied 291 patients who underwent infrainguinal interventions and noted that diabetics had a primary patency rate of $53 \pm 4 \%$, compared to $71 \pm 4 \%$ for non-diabetics. On the other hand, O'Connor et al. ${ }^{22}$ did not find any relationship between HbA1c and disease severity or the need for re-interventions in diabetics with limb-threatening ischemia. While acknowledging the lack of strong evidence in this area, the American Diabetes Association and American College of Cardiology/American Heart Association (ACC/AHA) nevertheless recommend glycemic control (goal HbA1c <7\%) in patients with PAD.

The association between glycemic control and outcomes has been more extensively studied in the setting of coronary artery balloon angioplasty and stent placement. ${ }^{11,12,22-24}$ Both pre-procedural $\mathrm{HbA} 1 \mathrm{c}$ and fasting blood glucose have been evaluated as predictors of outcomes with mixed results. While Lemesle et al. ${ }^{23}$ did not find any correlation between major adverse cardiovascular events (MACE) and $\mathrm{HbA1c}$, others ${ }^{11,12}$ have reported contradictory results. The association between pre-procedural blood glucose and PCI outcomes has been somewhat more conclusive. Marfella et al. ${ }^{24}$ and Nusca et al. ${ }^{25}$ have reported that pre-procedure hyperglycemia was a predictor of long-term outcomes, including restenosis. Marfella ${ }^{24}$ examined the effects of peri-procedural intensive glucose control during PCI on restenosis rates in hyperglycemic patients (glucose $>140 \mathrm{mg} / \mathrm{dL}$ ) presenting with ST-segment myocardial infarction (STEMI). Patients were randomized to intensive glucose control (target glucose 80-140 mg/dL) or conventional control (glucose 180-200 $\mathrm{mg} / \mathrm{dL}$ ) for 24 hours after PCI. Both groups received conventional therapy after the first 24 hours. At follow-up angiography at 6 months, restenosis rates were significantly higher in the conventional control group ( $46 \%$ vs $24 \%, p<0.001)$. In another prospective analysis, Nusca et al. ${ }^{25}$ studied the effect of peri-procedural blood glucose levels on short- and longterm outcomes in 572 patients undergoing elective PCI. Patients were categorized into four groups: hypoglycemia ( $\$ 80 \mathrm{mg} / \mathrm{dL}$ ), normoglycemia (81-109 mg/dL), mild hyperglycemia $(110-125 \mathrm{mg} / \mathrm{dL}$ ) and hyperglycemia ( $\geq 126 \mathrm{mg} / \mathrm{dL}$ ). After a mean follow-up of $15 \pm 8$ months, they noted an increased incidence of restenosis and target vessel revascularization in the hypo- and hyperglycemia groups compared to those with normoglycemia ( $p$ for trend $<0.001)$.

We noted an almost fivefold increase in primary patency at 1 year for patients in the lowest quartile of FBG compared to those in the highest quartile of FBG. It is noteworthy that the association remained significant even when baseline insulin use was taken into account. This 
finding suggests that glycemic control at the time of the intervention may be a better predictor of primary patency than overall glycemic control in the months preceding intervention, which is reflected by measurement of HbA1c. ${ }^{13,24,25}$

The increased rate of target lesion failure among patients with diabetes has been attributed to numerous physiological mechanisms, including accelerated neointimal hyperplasia, impaired vessel remodeling, exaggerated thrombus formation, and persistent endothelial dysfunction. ${ }^{26,27}$ Although both HbA1c and FBG are measures of glycemic control, the FBG value at the time of angioplasty may be a better marker for acute glycemic control, suggesting that the acute metabolic milieu ${ }^{13,24}$ may play a significant role in subsequent restenosis. Several mechanisms have been proposed to link peri-procedural glycemic control and adverse outcomes after angioplasty. ${ }^{24,28,29,30}$ For example, acutely elevated glucose concentrations may promote inflammatory cell recruitment and stimulate smooth muscle proliferation and abnormal matrix production. In addition, hyperglycemia may inactivate endothelium-derived relaxing factor, which in turn inhibits smooth muscle proliferation. Hyperglycemia itself could affect the expression of several growth factors, such as basic fibroblast growth factor and transforming growth factor-alpha, which induce the proliferation of smooth muscle cells and extracellular matrix synthesis. ${ }^{31}$ Marfella et al. ${ }^{24}$ have proposed that tighter pre-procedural glycemic control is associated with a reduction in inflammatory cytokines (C-reactive protein and tumor necrosis factor-a), oxidative stress, and recovered endothelial function. Reduced oxidative stress may improve endothelial function by increasing the availability of free and active nitrous oxide. ${ }^{24}$ Acute hyperglycemia is also associated with increased levels of monocyte chemoattractantprotein-1, which has been linked to restenosis. ${ }^{24,32}$ There is also some evidence that shortterm hyperglycemia has an impact on thrombus formation. Gresele et al. ${ }^{33}$ studied platelet activation in diabetic patients with controlled hyperglycemia during glucose clamping at euglycemic and hyperglycemic levels. They noted that there was a greater degree of platelet activation during hyperglycemia during in vitro exposure to high shear stress conditions. Therefore, it is possible that abnormalities in inflammatory response, oxidative stress, and endothelial and platelet function at the time of angioplasty-induced vessel injury may acutely influence the subsequent risk of restenosis. Over the long-term, poor glycemic control has also been linked to poor healing of diabetic ulcers. ${ }^{34}$ This could also contribute to the higher rates of amputations that have been noted by some studies. ${ }^{21}$

Our finding that FBG at the time of balloon angioplasty predicts loss of primary patency also raises the question of whether intensive glycemic control at the time of intervention could improve vessel patency after angioplasty. Although these observational studies show an association between pre-procedure glycemic control and patency, a randomized trial would be necessary to determine whether improved peri-procedural glycemic control could reduce rates of target lesion failure and possibly improve overall limb salvage rates among patients with diabetes and CLI.

\section{Limitations}

This was a single-center, retrospective analysis. Prospective, dedicated collection of fasting blood glucose immediately pre-procedure would best reflect the glycemic environment at the 
time of balloon angioplasty. Although we were careful to identify pre-procedural fasting blood glucose values, it is possible that glucose at the time of balloon angioplasty differed slightly from pre-procedural blood values. We also do not have data on changes in glycemic control in the months following intervention. The optimal duration of intensive glycemic control after intervention is uncertain. This study also represents the experience of a single academic institution and therefore may not be generalizable to all clinical settings. Despite these limitations, the association of pre-procedure glucose and loss of primary patency remained robust despite multivariable analysis, and we also found a significant doseresponse association of FBG and patency.

\section{Conclusion}

High fasting blood glucose at the time of infrapopliteal balloon angioplasty is associated with significantly decreased primary patency. High FBG may also be a risk factor for subsequent amputation or need for surgical revascularization among patients with CLI. Intensive peri-procedural glucose control may improve outcomes of balloon angioplasty, and this approach should be studied in a randomized trial.

\section{Acknowledgments}

This project was supported in part by the National Center for Advancing Translational Sciences, National Institutes of Health, through grant number UL1 TR 000002 and linked award TL1 TR 000133.

\section{References}

1. Marso SP, Hiatt WR. Peripheral arterial disease in patients with diabetes. J Am Coll Cardiol. 2006; 47:921-929. [PubMed: 16516072]

2. Armstrong EJ, Rutledge JC, Rogers JH. Coronary artery revascularization in patients with diabetes mellitus. Circulation. 2013; 128:1675-1685. [PubMed: 24100481]

3. Jude EB, Oyibo SO, Chalmers N, et al. Peripheral arterial disease in diabetic and nondiabetic patients: a comparison of severity and outcome. Diabetes Care. 2001; 24:1433-1437. [PubMed: 11473082]

4. Faglia E, Favales F, Quarantiello A, et al. Angiographic evaluation of peripheral arterial occlusive disease and its role as a prognostic determinant for major amputation in diabetic subjects with foot ulcers. Diabetes Care. 1998; 21:625-630. [PubMed: 9571354]

5. Bild DE, Selby JV, Sinnock P, et al. Lower extremity amputation in people with diabetes: epidemiology and prevention. Diabetes Care. 1989; 12:24-31. [PubMed: 2714164]

6. DeRubertis BG, Pierce M, Ryer EJ, et al. Reduced primary patency rate in diabetic patients after percutaneous intervention results from more frequent presentation with limb-threatening ischemia. $\mathrm{J}$ Vasc Surg. 2008; 47:101-108. [PubMed: 18178459]

7. Abularrage CJ, Conrad MF, Hackney LA, et al. Long-term outcomes of diabetic patients undergoing endovascular infrainguinal interventions. J Vasc Surg. 2010; 52:314-322. [PubMed: 20591601]

8. The Action to Control Cardiovascular Risk in Diabetes Study Group. Effects of intensive glucose lowering in type 2 diabetes. N Engl J Med. 2008; 358:2545-2559. [PubMed: 18539917]

9. The ADVANCE Collaborative Group. Intensive glucose control and vascular outcomes in patients with type 2 diabetes. N Engl J Med. 2008; 358:2560-2572. [PubMed: 18539916]

10. Stratton IM, Adler AI, Neil HA, et al. Association of glycaemia with macrovascular and microvascular complications of type 2 diabetes (UKPDS 35): prospective observational study. BMJ. 2000; 321:405-412. [PubMed: 10938048] 
11. Ueda H, Mitsusada N, Harimoto K, et al. Glycosylated hemoglobin is a predictor of major adverse cardiac events after drug-eluting stent implantation in patients with diabetes mellitus. Cardiology. 2010; 116:51-57. [PubMed: 20453503]

12. Corpus RA, George PB, House JA, et al. Optimal glycemic control is associated with a lower rate of target vessel revascularization in treated type II diabetic patients undergoing elective percutaneous coronary intervention. J Am Coll Cardiol. 2004; 43:8-14. [PubMed: 14715174]

13. Lindsay J, Sharma AK, Canos D, et al. Preprocedure hyperglycemia is more strongly associated with restenosis in diabetic patients after percutaneous coronary intervention than is hemoglobin A1C. Cardiovasc Revasc Med. 2007; 8:15-20. [PubMed: 17293264]

14. McCoach CE, Armstrong EJ, Singh S, et al. Gender-related variation in the clinical presentation and outcomes of critical limb ischemia. Vasc Med. 2013; 18:19-26. [PubMed: 23439776]

15. Rutherford RB, Baker JD, Ernst C, et al. Recommended standards for reports dealing with lower extremity ischemia: Revised version. J Vasc Surg. 1997; 26:517-538. [PubMed: 9308598]

16. Norgren L, Hiatt WR, Dormandy JA, et al. Inter-Society Consensus for the Management of Peripheral Arterial Disease (TASC II). J Vasc Surg. 2007; 45:S5-S67. [PubMed: 17223489]

17. Conte MS. Understanding objective performance goals for critical limb ischemia trials. Semin Vasc Surg. 2010; 23:129-137. [PubMed: 20826289]

18. Selvin E, Wattanakit K, Steffes MW, et al. HbA1c and peripheral arterial disease in diabetes: the Atherosclerosis Risk in Communities study. Diabetes Care. 2006; 29:877-882. [PubMed: $16567831]$

19. Collins TC, Beyth RJ, Nelson DB, et al. Process of care and outcomes in patients with peripheral arterial disease. J Gen Intern Med. 2007; 22:942-948. [PubMed: 17453264]

20. O’Sullivan CJ, Hynes N, Mahendran B, et al. Haemoglobin A1c (HbA1c) in non-diabetic and diabetic vascular patients. Is HbA1c an independent risk factor and predictor of adverse outcome? Eur J Vasc Endovasc Surg. 2006; 32:188-197. [PubMed: 16580235]

21. Takahara M, Kaneto H, Iida $\mathrm{O}$, et al. The influence of glycemic control on the prognosis of Japanese patients undergoing percutaneous transluminal angioplasty for critical limb ischemia. Diabetes Care. 2010; 33:2538-2542. [PubMed: 20843974]

22. O'Connor DJ, Gargiulo NJ 3rd, Jang J. Hemoglobin A1c as a measure of disease severity and outcome in limb threatening ischemia. J Surg Res. 2012; 174:29-32. [PubMed: 21920547]

23. Lemesle G, Bonello L, de Labriolle A, et al. Prognostic value of hemoglobin A1C levels in patients with diabetes mellitus undergoing percutaneous coronary intervention with stent implantation. Am J Cardiol. 2009; 104:41-45. [PubMed: 19576319]

24. Marfella R, Sasso FC, Siniscalchi M, et al. Peri-procedural tight glycemic control during early percutaneous coronary intervention is associated with a lower rate of in-stent restenosis in patients with acute ST-elevation myocardial infarction. J Clin Endocrinol Metab. 2012; 97:2862-2871. [PubMed: 22639289]

25. Nusca A, Patti G, Marino F, et al. Prognostic role of pre-procedural glucose levels on short- and long-term outcome in patients undergoing percutaneous coronary revascularization. Catheter Cardiovasc Interv. 2012; 80:377-384. [PubMed: 21681905]

26. Kornowski R, Fuchs S. Optimization of glycemic control and restenosis prevention in diabetic patients undergoing percutaneous coronary interventions. J Am Coll Cardiol. 2004; 43:15-17. [PubMed: 14715175]

27. Aronson D, Bloomgarden Z, Rayfield EJ. Potential mechanisms promoting restenosis in diabetic patients. J Am Coll Cardiol. 1996; 27:528-535. [PubMed: 8606261]

28. McClain DA, Paterson AJ, Roos MD, et al. Glucose and glucosamine regulate growth factor gene expression in vascular smooth muscle cells. Proc Natl Acad Sci U S A. 1992; 89:8150-8154. [PubMed: 1518840]

29. Lindner V, Reidy MA. Proliferation of smooth muscle cells after vascular injury is inhibited by an antibody against basic fibroblast growth factor. Proc Natl Acad Sci U S A. 1991; 88:3739-3743. [PubMed: 2023924]

30. Kornowski R, Mintz GS, Kent KM, et al. Increased restenosis in diabetes mellitus after coronary interventions is due to exaggerated intimal hyperplasia. A serial intravascular ultrasound study. Circulation. 1997; 95:1366-1369. [PubMed: 9118501] 
31. Nakamura N, Ueno Y, Tsuchiyama Y, et al. Isolated post-challenge hyperglycemia in patients with normal fasting glucose concentration exaggerates neointimal hyperplasia after coronary stent implantation. Circ J. 2003; 67:61-67. [PubMed: 12520154]

32. Cipollone F, Marini M, Fazia M, et al. Elevated circulating levels of monocyte chemoattractant protein-1 in patients with restenosis after coronary angioplasty. Arterioscler Thromb Vasc Biol. 2001; 21:327-334. [PubMed: 11231910]

33. Gresele P, Guglielmini G, De Angelis M, et al. Acute, short-term hyperglycemia enhances shear stress-induced platelet activation in patients with type II diabetes mellitus. J Am Coll Cardiol. 2003; 41:1013-1020. [PubMed: 12651051]

34. Markuson M, Hanson D, Anderson J, et al. The relationship between hemoglobin A1c values and healing time for lower extremity ulcers in individuals with diabetes. Adv Skin Wound Care. 2009; 22:365-372. [PubMed: 19638800] 


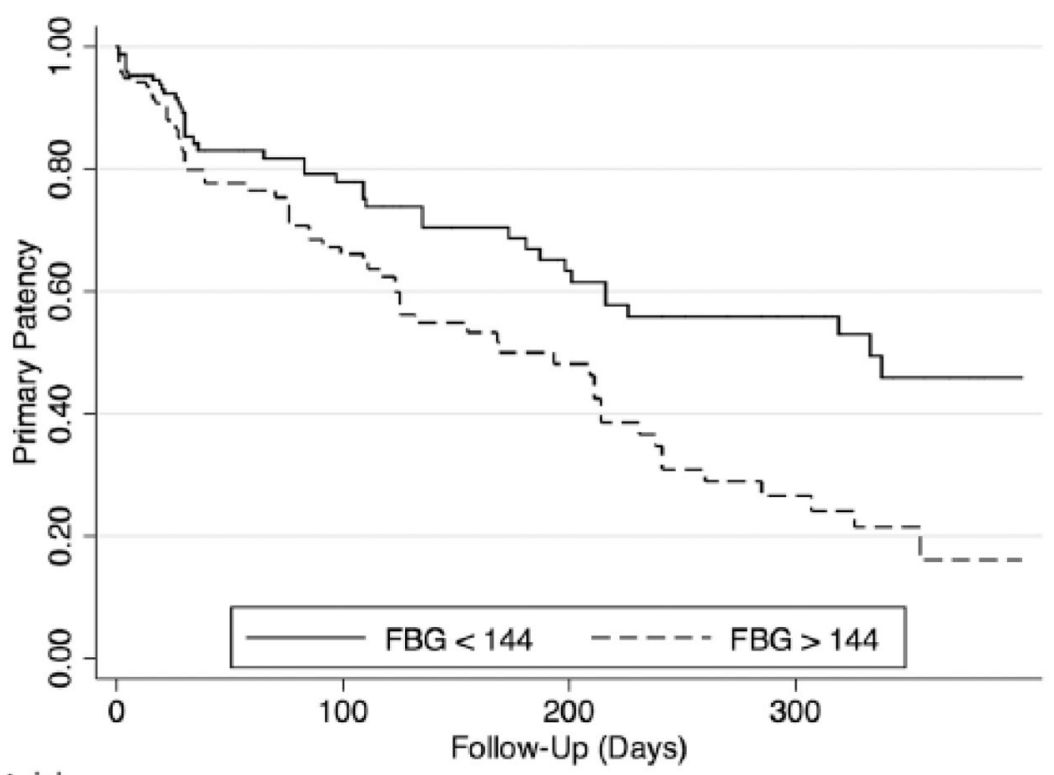

Number at risk

FBG $<144152$

$\mathrm{FBG}>144157$

58

34

23

$56 \quad 26$

11

Figure 1.

Rates of primary patency among patients with diabetes undergoing infrapopliteal balloon angioplasty. A fasting blood glucose $(\mathrm{FBG})>144 \mathrm{mg} / \mathrm{dL}$ at the time of the procedure was associated with significantly decreased primary patency. 


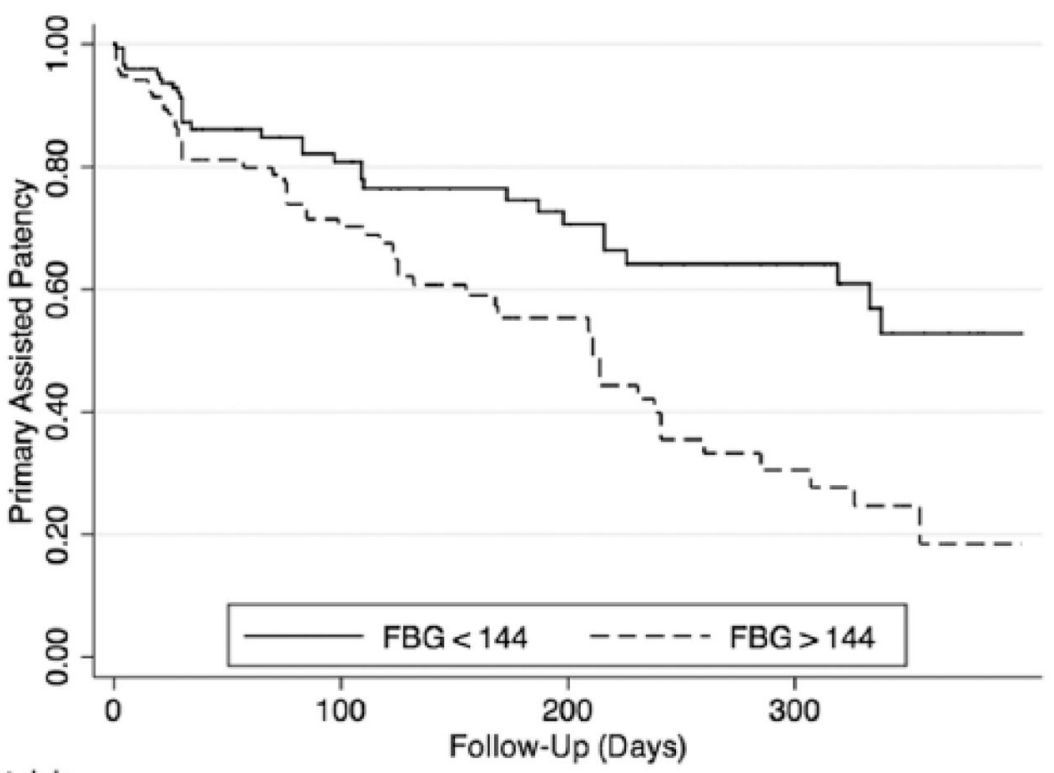

Number at risk

$\mathrm{FBG}<144152$

$\mathrm{FBG}>144157$

58

56

34

23

11

Figure 2.

Primary assisted patency (FBG, fasting blood glucose). 


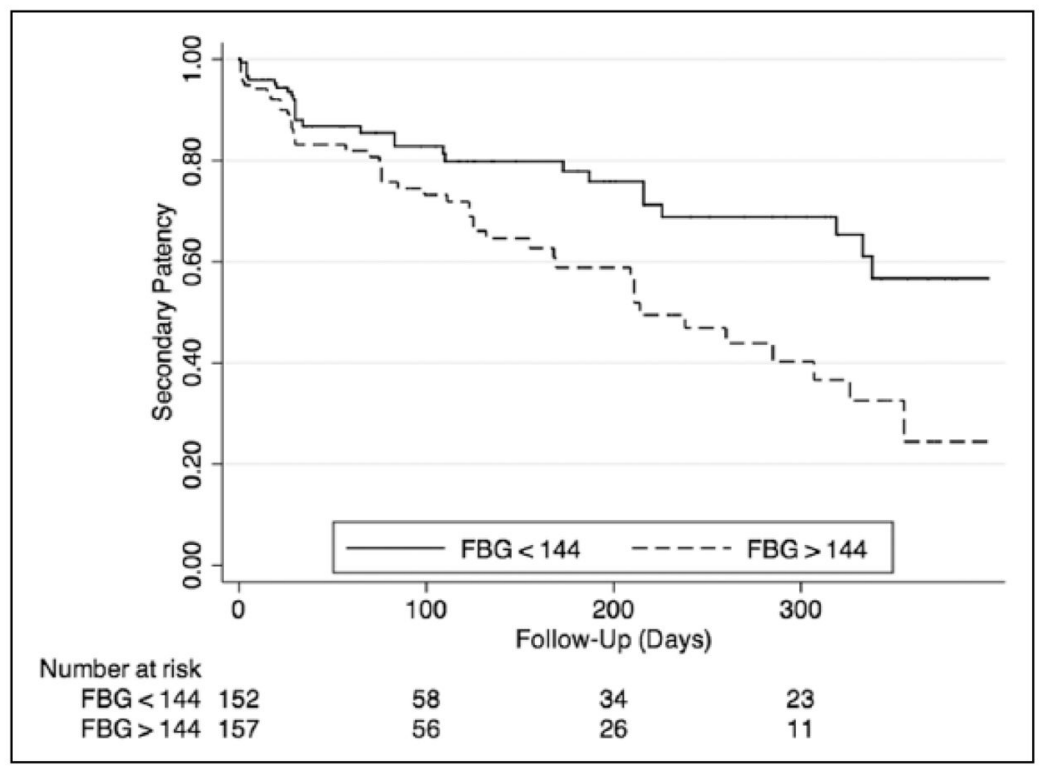

Figure 3.

Secondary patency (FBG, fasting blood glucose). 


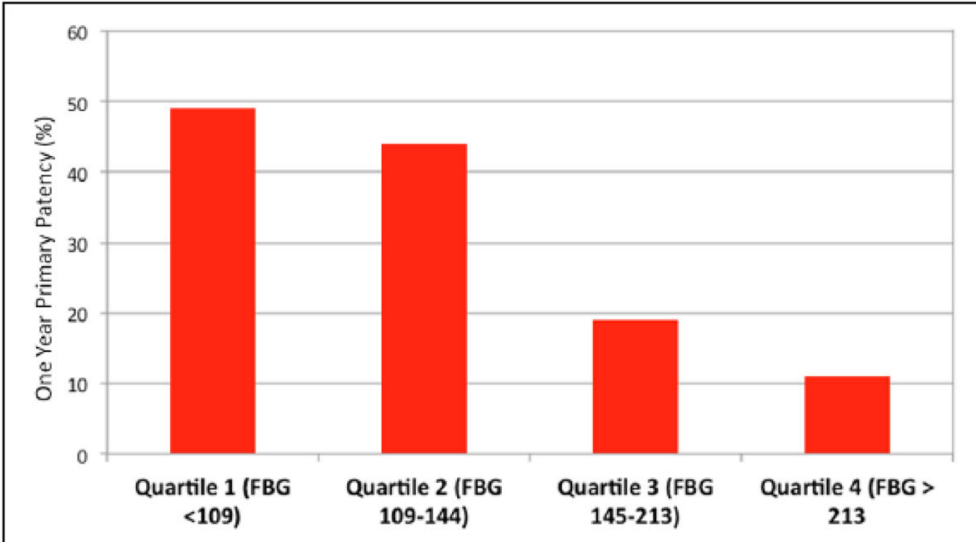

Figure 4.

Primary patency after balloon angioplasty among quartiles (FBG, fasting blood glucose). 


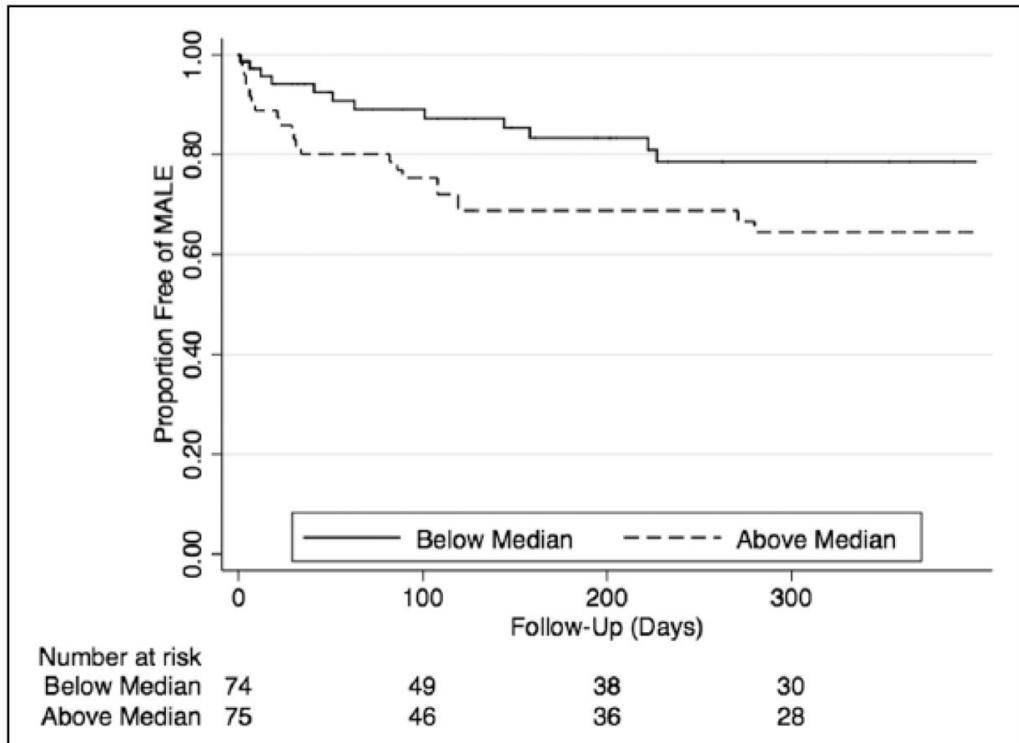

Figure 5.

Major adverse limb events (MALE). 


\section{Table 1}

\section{Baseline patient characteristics.}

\begin{tabular}{|c|c|c|c|}
\hline Variable & Fasting glucose $<144(n=74)$ & Fasting glucose $\geq 144(n=75)$ & $p$-value \\
\hline Age, years & $69.6 \pm 10.6$ & $67.3 \pm 11.6$ & 0.2 \\
\hline Male (\%) & $53(72)$ & $48(64)$ & 0.3 \\
\hline $\mathrm{BMI}, \mathrm{kg} / \mathrm{m}^{2}$ & $29.0 \pm 6.9$ & $31.0 \pm 8.3$ & 0.1 \\
\hline Tobacco, former or current $(\%)$ & $35(48)$ & $39(53)$ & 0.6 \\
\hline Tobacco, current $(\%)$ & $8(11)$ & $11(15)$ & 0.5 \\
\hline $\mathrm{CHF}(\%)$ & $21(29)$ & $27(36)$ & 0.3 \\
\hline Insulin-requiring DM (\%) & $37(50)$ & $55(73)$ & 0.003 \\
\hline $\mathrm{GFR}, \mathrm{ml} / \mathrm{min}$ & $51 \pm 34$ & $60 \pm 41$ & 0.2 \\
\hline $\operatorname{HTN}(\%)$ & $67(91)$ & $64(85)$ & 0.3 \\
\hline $\mathrm{CAD}(\%)$ & $40(55)$ & $41(55)$ & 0.9 \\
\hline History of MI (\%) & $15(20)$ & $17(23)$ & 0.7 \\
\hline History of stroke/TIA (\%) & $16(22)$ & $17(23)$ & 0.9 \\
\hline $\operatorname{COPD}(\%)$ & $4(5)$ & $9(12)$ & 0.2 \\
\hline History of AAA (\%) & $0(0)$ & $0(0)$ & 1.0 \\
\hline History of carotid stenosis (\%) & $10(15)$ & $8(11)$ & 0.5 \\
\hline History of contralateral amputation (\%) & $12(16)$ & $16(21)$ & 0.4 \\
\hline $\mathrm{LDL}, \mathrm{mg} / \mathrm{dl}$ & $76 \pm 27$ & $83 \pm 35$ & 0.3 \\
\hline $\mathrm{HbA1c}, \%$ & $7.6 \pm 2.0$ & $8.4 \pm 1.9$ & 0.04 \\
\hline Aspirin $(\%)$ & $69(93)$ & $72(96)$ & 0.5 \\
\hline Statin $(\%)$ & $49(66)$ & $53(71)$ & 0.6 \\
\hline Beta-blocker $(\%)$ & $41(55)$ & $46(61)$ & 0.5 \\
\hline Clopidogrel (\%) & $27(36)$ & $30(40)$ & 0.7 \\
\hline ACE inhibitor (\%) & $44(59)$ & $42(56)$ & 0.7 \\
\hline Rutherford score & & & 0.3 \\
\hline 4 & $13(18)$ & $7(9)$ & \\
\hline 5 & $49(66)$ & $55(73)$ & \\
\hline 6 & $12(16)$ & $13(17)$ & \\
\hline Ankle-brachial index & $0.34 \pm 0.1$ & $0.31 \pm 0.1$ & 0.7 \\
\hline Toe-brachial index & $0.29 \pm 0.1$ & $0.29 \pm 0.1$ & 0.6 \\
\hline
\end{tabular}

BMI, body mass index; CHF, congestive heart failure; DM, diabetes; GFR, glomerular filtration rate; HTN, hypertension; CAD, coronary artery disease; MI, myocardial infarction; TIA, transient ischemic attack; COPD, chronic obstructive pulmonary disease; AAA, abdominal aortic aneurysm; LDL, low density lipoprotein; HbA1c, hemoglobin A1c; ACE, angiotensin converting enzyme. 
Table 2

Lesion and procedural characteristics.

\begin{tabular}{llll}
\hline Variable & Fasting glucose $<\mathbf{1 4 4}(\boldsymbol{n}=\mathbf{1 5 2})$ & Fasting glucose $\mathbf{\geq 1 4 4}(\boldsymbol{n}=\mathbf{1 5 7})$ & $\boldsymbol{p}$-value \\
\hline Lesion location & & & 0.6 \\
$\quad$ Tibioperoneal trunk & $41(27)$ & $34(22)$ & \\
$\quad$ Peroneal & $26(17)$ & $31(20)$ & \\
$\quad$ Anterior tibial & $49(32)$ & $59(37)$ & \\
$\quad$ Posterior tibial & $36(24)$ & $33(21)$ & 0.9 \\
Mean lesion length, mm & $92 \pm 74$ & $94 \pm 68$ & 0.5 \\
Pre-procedure stenosis, \% & $82 \pm 24$ & $84 \pm 21$ & 0.6 \\
Vessel diameter, mm & $2.6 \pm 0.6$ & $2.7 \pm 0.6$ & 0.8 \\
Chronic occlusion & $59(41)$ & $63(40)$ & 0.1 \\
Calcification & & & \\
$\quad$ Mild-moderate & $109(74)$ & $127(83)$ & 0.3 \\
$\quad$ Severe & $37(25)$ & $27(18)$ & 0.2 \\
Procedural success, \% & $140(94)$ & $143(91)$ & 0.3 \\
Post-procedure ABI, 30 days & $0.79 \pm 0.1$ & $0.73 \pm 0.1$ \\
Post-procedure TBI, 30 days & $0.4 \pm 0.2$ & $0.4 \pm 0.2$ & \\
\hline
\end{tabular}

ABI, ankle-brachial pressure index; TBI, toe-brachial index. 
Table 3

Univariate and multivariate associations of primary patency after infrapopliteal balloon angioplasty.

\begin{tabular}{lcc}
\hline Variable & Hazard ratio (95\% CI) & Adjusted hazard ratio (95\% CI) \\
\hline Fasting blood glucose & $1.8(1.2-2.8)$ & $1.8(1.2-2.8)$ \\
Insulin use & $1.2(0.8-1.9)$ & $1.2(0.7-2.0)$ \\
Lesion length & $1.02(1.01-1.05)$ & $1.1(1.1-1.2)$ \\
Chronic total occlusion & $1.5(1.05-2.3)$ & $1.4(1.0-2.2)$ \\
Statin use & $0.8(0.5-1.4)$ & $0.8(0.5-1.4)$ \\
Glomerular filtration rate & $1.0(0.99-1.07)$ & $0.95(0.90-0.99)$ \\
Dialysis & $0.9(0.5-1.5)$ & $0.94(0.5-1.7)$ \\
\hline
\end{tabular}




\section{Table 4}

Univariate and multivariate associations of major adverse limb events after infrapopliteal balloon angioplasty.

\begin{tabular}{lll}
\hline Variable & Hazard ratio $(95 \%$ CI $)$ & Adjusted hazard ratio $(95 \%$ CI $)$ \\
\hline Fasting blood glucose & $2.0(1.0-3.9)$ & $2.3(1.1-5.2)$ \\
Insulin use & $1.4(0.7-3.0)$ & $1.3(0.6-3.0)$ \\
Lesion length & $1.0(0.9-1.1)$ & $1.0(0.9-1.1)$ \\
Chronic total occlusion & $1.9(1.0-3.8)$ & $2.0(0.9-4.1)$ \\
Statin use & $0.7(0.3-1.4)$ & $0.7(0.3-1.6)$ \\
Glomerular filtration rate & $0.9(0.9-1.0)$ & $1.0(0.9-1.1)$ \\
Dialysis & $1.6(0.8-3.2)$ & $1.8(0.7-4.7)$ \\
\hline
\end{tabular}

